\title{
Avaliação das características químicas, físico-químicas e da rotulagem de compostos líquidos prontos para consumo
}

\author{
Francisca Imilena Pereira de Oliveira ${ }^{1}$, José Maria Correia da Costa ${ }^{2}$, \\ Érica Milô de Freitas Felipe Rocha ${ }^{3}$
}

Nos últimos anos tem ocorrido uma crescente demanda pelo consumo de bebidas energéticas, composto líquido pronto para consumo, porém, são escassas as publicações sobre a composição destas bebidas. Desse modo, o objetivo dessa pesquisa foi elaborar a caracterização química e físico-química bem como implementar a avaliação da rotulagem de quatro marcas de bebidas energéticas comerciais e disponibilizar esta informação ao consumidor. Foram avaliados $\mathrm{pH}$, acidez, condutividade elétrica, viscosidade, concentração salina, sólidos solúveis totais, ácido ascórbico, açúcares redutores e não redutores, açúcares totais, sólidos totais e cafeína. A análise da rotulagem teve por base os registros da legislação vigente. Os resultados das análises químicas e físicoquímicas apresentaram diferenças significantes ao nível de 5\% em pelo menos uma das amostras estudadas. Vale ressaltar que a quantidade de cafeína encontrada, nos produtos de todas as marcas é menor que o limite máximo permitido pela legislação, que estabelece um valor máximo de $35 \mathrm{mg} / 100 \mathrm{~mL}$. Com relação à análise dos conteúdos dos rótulos, as marcas analisadas não atenderam plenamente a totalidade dos requisitos exigidos pela legislação.

Palavras-chave: bebidas energizantes, composição, composto líquido pronto para consumo, ANVISA.

\section{Evaluation of chemical and physical-chemical characteristics and label application of ready-to-drink liquid composites}

In recent years a growing demand has occurred in the consumption of energizing drinks, like Liquid Composite Ready for Consumption. There is however is a lack of literature regarding the composition of these beverages. Thus, the goal of this research was to perform a chemical and physico-chemical characterization of four commercially available drinks as well as to evaluate the labeling, making this general information accessible to consumers uses. $\mathrm{pH}$, acidity, electrical conductivity, viscosity, salt concentration, total soluble solids, ascorbic acid, reducing and non reducing sugars, total sugars, total solids and caffeine were determined. The label analysis was based on current legislation recommendations. The results of chemical and physicochemical analyses showed significant differences at the $5 \%$ probability level of at least one of the samples. It is noteworthy that in all commercial brands, the amount of caffeine was below the maximum allowed, which establishes a maximum of $35 \mathrm{mg} / 100 \mathrm{~mL}$. Regarding the label analysis, the brands tested did not fully met all the requirements stated by the pertinent laws.

Key-words: energizing drinks, composition, liquid composite ready for consumption, ANVISA.

\footnotetext{
${ }^{1}$ Mestre em Engenharia Química, Universidade Federal do Ceará (UFC).

2 Professor Doutor do Departamento de Tecnologia de Alimentos, Universidade Federal do Ceará (UFC).

Correspondência: Av. Mister Hull, 2977, Campus do Pici, CEP 60356-000, Fortaleza(CE). E-mail: correia@ufc.br

${ }^{3}$ Doutoranda do Programa de Ciência e Tecnologia de Alimentos, Departamento de Tecnologia de Alimentos, Universidade Federal do Ceará (UFC).
} 


\section{INTRODUÇÃO}

Há alguns anos o mercado está sendo invadido por bebidas denominadas energéticas, que segundo seus produtores foram criadas para incrementar a resistência física, proporcionar reações mais rápidas e maior concentração, aumentar o estado de alerta mental, evitar o sono, proporcionar sensação de bem estar, estimular o metabolismo e ajudar a eliminar substâncias nocivas ao corpo ${ }^{[1]}$.

Esses repositores energéticos são produtos formulados com nutrientes que permitam o alcance e/ou manutenção do nível apropriado de energia para atletas. Nestes produtos, segundo a Agência Nacional de Vigilância Sanitária (ANVISA), os carboidratos devem constituir, no mínimo, 90\% dos nutrientes energéticos presentes na formulação e, opcionalmente, estes produtos podem conter vitaminas e/ou minerais [2].

Nesta busca do sucesso esportivo de alto nível, treinadores, nutricionistas, médicos e cientistas têm lançado mão de inúmeros recursos ergogênicos no intuito de potencializar a performance atlética ou atenuar os mecanismos geradores da fadiga de seus atletas [3,4,5] .

Estes produtos energéticos são classificados como Composto Líquido Pronto para Consumo que se caracterizam por serem produtos que contém como ingrediente(s) principal(is): inositol, glucoronolactona e/ou taurina e/ou cafeína, podendo ser adicionado de vitaminas e/ou minerais até $100 \%$ da Ingestão Diária Recomendada (IDR) na porção do produto. Pode ser adicionado de outro(s) ingrediente(s), desde que não descaracterize $(\mathrm{m})$ o produto [0].

Acredita-se que a cafeína possua mecanismos de ação central e periférica que podem desencadear importantes alterações metabólicas e fisiológicas, as quais parecem melhorar a performance atlética [7,8,9]. Registra-se que as metilxantinas como a cafeína induzem a aumentos na dose-resposta da atividade locomotora em animais [10]. Todavia o seu efeito ergogênico é ainda bastante controverso, visto que aparentemente outros mecanismos podem estar associados à sua ação, melhorando a performance em diferentes tipos de exercícios ${ }^{[11]}$.

O risco potencial do consumo das bebidas energéticas pode ser aumentado pelo uso concomitante com outras substâncias, como o álcool. Este fato pode causar uma intoxicação e, possivelmente, provocar convulsões, taquicardia, arritmias, parada cardíaca ou morte súbita. Estas bebidas energéticas parecem retardar os efeitos depressores do álcool e, este fato, tem levado a um aumento no consumo de bebidas alcoólicas [12,13]. Sendo este um problema identificado especialmente entre os jovens, considerados os principais prejudicados, pois tem livre acesso a estas bebidas nos locais onde se reúnem para dançar, em clubes, bares, academias, centros esportivos, e concertos musicais [14], onde estas são vendidas separadamente ou até juntamente com bebidas alcoólicas.

Para garantir a segurança do consumidor que opta por essas bebidas, é necessário que os níveis dos micronutrientes atendam ao registrado na IDR, além de estarem em acordo com o declarado no rótulo pelos fabricantes. Em função do exposto e, considerando a escassez de publicações relacionadas às bebidas energéticas, este trabalho teve como objetivo identificar a composição destas bebidas, por meio da análise de suas características químicas e físico-químicas. $\mathrm{O}$ trabalho visou também a análise da rotulagem.

\section{MATERIAL E MÉTODOS}

Foram analisadas quatro marcas comerciais de bebidas energéticas, todas produzidas por distintos fabricantes nacionais e, adquiridas em grandes redes de supermercados da cidade de Fortaleza (CE). De cada marca foram adquiridos $500 \mathrm{~mL}$ de bebida energética em embalagens metálicas (embalagem comercial e dentro do prazo de validade). As marcas estudadas foram designadas de A, B, C e D, objetivando manter o sigilo em relação aos seus fabricantes.

As determinações químicas e físico-químicas, realizadas em triplicatas, nas amostras das diferentes marcas de bebidas energéticas foram efetuadas no Laboratório de Controle de Qualidade do Departamento de Tecnologia em Alimentos da Universidade Federal do Ceará. $\mathrm{O}$ pH foi determinado através de um potenciômetro digital; acidez titulável pelo método de "titulação com solução de hidróxido 0,1 molar" e expressa em g de ácido cítrico/100 mL; as análises de sólidos solúveis totais ( $\mathrm{em}^{\circ} \mathrm{Brix}$ ), através de refratômetro Analytik Jena ${ }^{\circledR}$ a $20^{\circ} \mathrm{C}$; açúcares redutores - método de Lane-Eynon e os resultados expressos em termos de percentual de glicose e açúcares não redutores. Os referidos procedimentos seguiram as normas do Instituto Adolfo Lutz [15]. Os resultados foram expressos em percentual de sacarose; os açúcares totais foram obtidos pelo somatório dos açúcares redutores e não redutores; o conteúdo de ácido ascórbico foi determinado através do método 
titulométrico, baseado na redução do indicador 2,6diclorofenolindofenol pelo ácido ascórbico [16]; condutividade elétrica, por condutivímetro marca Schott ${ }^{\mathbb{R}}$ e a concentração salina foi obtida pelo mesmo aparelho e expressa em $\mathrm{mg} / \mathrm{L}$; a viscosidade foi realizada por meio de um viscosímetro capilar, também da marca Schott ${ }^{\mathbb{R}}$; os sólidos totais foram determinados pelo método gravimétrico, fundamentando-se na evaporação da água e substâncias voláteis e pesagem do extrato não volatizado e o teor de cafeína foi determinado por cromatografia líquida e os resultados foram expressos em mg/100 mL [17]. A análise da rotulagem das quatro marcas dos compostos líquidos prontos para o consumo contemplou os itens: apresentação das advertências obrigatórias, teor de cafeína, designação correta do produto, apresentação da expressão: "bebidas energéticas" (Resolução - RDC no 273/2005 [6]); denominação de venda e marca do produto, lista de ingredientes obrigatórios, conteúdos líquidos, identificação de origem, identificação de lote e prazo de validade, declaração da água na lista de ingredientes, declaração de aditivos alimentares na lista de ingredientes (Resolução - RDC no 259/2002 [18]; apresentação da rotulagem nutricional (Resolução RDC nㅇ 360/2003 [19].

Os resultados químicos e físico-químicos foram tratados estatisticamente por meio da Análise de variância (ANOVA) e aplicado o teste de Tukey $(p=$ $0,05)^{[20] .}$

\section{RESULTADOS E DISCUSSÃO}

$\mathrm{Na}$ Tabela 1 são apresentados os valores médios (acompanhados do desvio padrão) relativos aos parâmetros de $\mathrm{pH}$, acidez, condutividade, concentração salina e viscosidade das quatro bebidas energéticas analisadas.

Observa-se que a marca $\mathrm{A}$ apresentou as maiores concentrações de $\mathrm{pH}(3,11)$, acidez $(2,60 \%)$, condutividade elétrica $(1,87 \mu \mathrm{S} / \mathrm{cm})$, concentração salina $(771,33 \mathrm{mg} / \mathrm{L})$ e viscosidade $\left(6,35 \mathrm{~mm}^{2} / \mathrm{s}\right)$.

Os resultados da análise de variância de cada característica são apresentados nas Tabelas 1 e 2.

Note-se que na Tabela 2 é possível verificar os resultados obtidos para os parâmetros de sólidos totais, sólidos solúveis totais, açúcares redutores, açúcares não redutores, açúcares totais, vitamina $\mathrm{C}$ e cafeína. Verifica-se que há diferenças significativas entre as amostras para a totalidade das características analisadas.
Ainda de acordo com a Tabela 1 verifica-se que os valores de $\mathrm{pH}$ variaram entre 2,65 e 3,11, valores inferiores ao valor de 4,5, valor este, que delimita o crescimento de microrganismos e, de acordo com os dados obtido, é possível dizer que estas bebidas podem ser classificados como bebidas ácidas e, por conseguinte, de difícil ataque microbiano.

É importante, inicialmente, esclarecer a diferença entre o valor de acidez titulável e $\mathrm{pH}$, onde o primeiro, segundo Fontes [21], expressa a quantidade de ácido presente enquanto o $\mathrm{pH}$ expressa apenas o ácido dissociado na solução. Os valores de acidez variaram de $1,50 \%$ a $2,60 \%$ e a amostra $A$ revelou uma acidez aproximadamente duas vezes superior a obtida para a amostra C. As amostras C e D possuem valores de acidez relativamente próximos, que não apresentaram diferença estatisticamente significativa. É relevante ressaltar que a acidez é um parâmetro considerado de grande importância na qualidade e conservação destes produtos.

De acordo com Asenjo [22], a acidez do suco varia proporcionalmente ao conteúdo de vitamina $C$, esta variação embora direta, não é linear, o que indica a presença de outros ácidos. Pode-se perceber isto também em relação às bebidas energéticas, pois a amostra $\mathrm{C}$ apresentou o menor percentual de acidez e a menor quantidade de vitamina $\mathrm{C}$, porém por não ser linear, a amostra A que revelou o maior percentual de acidez foi a que apresentou a segunda menor quantidade de vitamina $\mathrm{C}$.

Em relação à análise de condutividade, parâmetro que expressa a capacidade do produto de conduzir corrente elétrica, é possível observar que as concentrações encontradas obedeceram à mesma ordem verificada para os parâmetros de concentração salina e acidez, demonstrando com isso que estas variáveis, quando realizado um acompanhamento regular da qualidade do produto, podem ser considerados parâmetros relevantes por fornecerem indícios de alteração do mesmo e ainda, indicar o surgimento de características corrosivas. Já em relação à viscosidade das bebidas, os teores apresentaram-se sob valores absolutos muito próximos. No entanto, todos diferem significativamente entre si.

Tendo por base os resultados obtidos para o parâmetro de sólidos totais apresentados (Tabela 2), observa-se que os mesmos variam entre 11,10 e $12,46 \%$, revelando também diferença significativa entre as amostras. Os resultados de sólidos solúveis totais, 
Tabela 1. Valores médios de pH, acidez, condutividade, concentração salina e viscosidade de quatro marcas de composto líquido pronto para consumo.

\begin{tabular}{lrrrr}
\hline \multirow{2}{*}{ Variáveis analisadas } & \multicolumn{4}{c}{ Marcas } \\
\cline { 2 - 5 } & \multicolumn{1}{c}{$\mathrm{A}$} & $\mathrm{B}$ & $\mathrm{C}$ & $\mathrm{D}$ \\
\hline $\mathrm{pH}$ & $3,11^{\mathrm{a}} \pm 0,00$ & $2,65^{\mathrm{c}} \pm 0,00$ & $2,79^{\mathrm{b}} \pm 0,00$ & $2,79^{\mathrm{b}} \pm 0,00$ \\
Acidez em ácido cítrico (\%) & $2,60^{\mathrm{a}} \pm 0,00$ & $2,13^{\mathrm{b}} \pm 0,06$ & $1,50^{\mathrm{c}} \pm 0,20$ & $1,73^{\mathrm{c}} \pm 0,06$ \\
Condutividade elétrica $(\mu \mathrm{S} / \mathrm{cm})$ & $1,87^{\mathrm{a}} \pm 0,00$ & $1,17^{\mathrm{b}} \pm 0,01$ & $1,11^{\mathrm{d}} \pm 0,01$ & $1,16^{\mathrm{c}} \pm 0,00$ \\
Concentração salina $(\mathrm{mg} / \mathrm{L})$ & $771,33^{\mathrm{a}} \pm 0,58$ & $84,33^{\mathrm{b}} \pm 0,58$ & $460,33^{\mathrm{d}} \pm 0,58$ & $476,00^{\mathrm{c}} \pm 0,00$ \\
Viscosidade $\left(\mathrm{mm}^{2} / \mathrm{s}\right)$ & $6,35^{\mathrm{a}} \pm 0,01$ & $6,29^{\mathrm{b}} \pm 0,01$ & $6,07^{\mathrm{d}} \pm 0,00$ & $6,22^{\mathrm{c}} \pm 0,01$
\end{tabular}

Valores médios numa mesma linha com letras minúsculas e iguais, não diferem significativamente entre si $(p=0,05)$, segundo o Teste de Tukey.

Tabela 2. Componentes físico-químicos das quatro marcas de composto líquido pronto para consumo.

\begin{tabular}{lcccc}
\hline \multirow{2}{*}{ Variáveis analisadas } & \multicolumn{4}{c}{ Marcas } \\
\cline { 2 - 5 } & $12,46^{\mathrm{a}} \pm 0,09$ & $12,46^{\mathrm{a}} \pm 0,13$ & $11,10^{\mathrm{b}} \pm 0,09$ & $12,23^{\mathrm{a}} \pm 0,01$ \\
\hline Sólidos totais (\%) & $12,63^{\mathrm{a}} \pm 0,01$ & $12,43^{\mathrm{b}} \pm 0,01$ & $11,40^{\mathrm{c}} \pm 0,00$ & $12,63^{\mathrm{a}} \pm 0,01$ \\
Sólidos solúveis totais ( ${ }^{\circ}$ Brix) & $4,28^{\mathrm{b}} \pm 0,27$ & $9,57^{\mathrm{a}} \pm 0,23$ & $9,00^{\mathrm{a}} \pm 0,00$ & $9,15^{\mathrm{a}} \pm 0,41$ \\
Açúcares redutores (\%) & $5,53^{\mathrm{a}} \pm 0,36$ & $0,91^{\mathrm{bc}} \pm 0,25$ & $0,12^{\mathrm{c}} \pm 0,04$ & $1,69^{\mathrm{b}} \pm 0,69$ \\
Açúcares não redutores $(\%)$ & $9,81^{\mathrm{bc}} \pm 0,37$ & $10,48^{\mathrm{ab}} \pm 0,02$ & $9,12^{\mathrm{c}} \pm 0,04$ & $10,83^{\mathrm{a}} \pm 0,45$ \\
Açúcares totais (\%) & $19,82^{\mathrm{c}} \pm 0,02$ & $38,67^{\mathrm{b}} \pm 0,01$ & $0,48^{\mathrm{d}} \pm 0,01$ & $57,19^{\mathrm{a}} \pm 0,35$ \\
Vitamina C (mg/100 ml) & $25,62^{\mathrm{b}} \pm 0,20$ & $11,33^{\mathrm{d}} \pm 0,11$ & $20,52^{\mathrm{c}} \pm 0,02$ & $30,24^{\mathrm{a}} \pm 0,03$ \\
Cafeína (mg/100 ml) & &
\end{tabular}

Valores médios numa mesma linha com letras minúsculas e iguais, não diferem (Teste de Tukey), significativamente entre si $(p=0,05)$.

também não apresentaram variações expressivas, fixando entre os valores 11,40 e $12,60{ }^{\circ}$ Brix. Segundo Chitarra e Alves [23], este parâmetro de sólidos solúveis totais representa concentração de substâncias dissolvidas no conteúdo celular, entre as quais destacam-se as vitaminas, pectinas, fenóis, ácidos orgânicos, pigmentos e, principalmente, os açúcares, podendo constituir cerca de 85 a 90\% deste parâmetro.

Em relação aos resultados da determinação de açúcares redutores (parâmetro que demonstra a potencialidade de fermentação do produto) verificou-se que a marca $\mathrm{A}$ apresentou um teor de 4,38\%, sendo em torno de cinquenta por cento inferior aos teores apresentados pelas marcas B, C e D que foram respectivamente de 9,56; 9,00 e 9,14\%. Sendo, consequentemente, observado o resultado inverso para a análise de açúcares não redutores.

Segundo Préstamo e Manzano [24], o uso de ácido ascórbico como antioxidante além de ser totalmente seguro para o consumo humano pode aumentar o teor de vitamina C. A declaração deste teor de vitamina $\mathrm{C}$ no rótulo de bebidas energéticas não é 
obrigatória, mas de acordo com a legislação o valor de IDR é $45 \mathrm{mg}$ [19]. Os resultados obtidos das análises, tendo por base as distintas marcas, mostram variação entre 19,82 e 57,19 mg/100 ml, sendo que a marca D foi a que apresentou o teor de vitamina $\mathrm{C}$ mais elevado, mostrando-se superior ao limite máximo permitido, ou seja, superior ao estabelecido [6] que admite a presença de vitaminas até $100 \%$ da IDR no produto a ser consumido.

Ainda tendo por base os dados da Tabela 2, as quantidades de cafeína nas amostras dos produtos prontos para o consumo variaram entre as marcas analisadas, sendo que os coeficientes de variação situaram-se entre $11,33 \%$ e 30,24\%, demonstrando, desta forma, que todas as marcas estão de acordo com o limite máximo permitido pelo Brasil que estabelece um valor de $35 \mathrm{mg} / \mathrm{mL}^{[6]}$.

A Tabela 3 reúne os dados relativos às analises envolvendo a rotulagem. Cabe lembrar que foram adotados parâmetros referentes à legislação vigente $[6,18,19]$.

Tabela 3. Análise da rotulagem de compostos líquidos prontos para o consumo.

\begin{tabular}{|c|c|c|c|c|}
\hline \multirow{2}{*}{ Parâmetros analisados } & \multicolumn{4}{|c|}{ Amostras } \\
\hline & A & $\mathrm{B}$ & $\mathrm{C}$ & $\mathrm{D}$ \\
\hline Apresentação das advertências obrigatórias & consta & consta & consta & consta \\
\hline Teor de cafeína & consta & consta & consta & consta \\
\hline Designação correta do produto & consta & consta & consta & consta \\
\hline Apresentação da expressão: "Bebidas energéticas" & não consta & consta & consta & consta \\
\hline Apresentação da rotulagem nutricional & consta & consta & consta & consta \\
\hline Denominação de venda e marca do produto & consta & consta & consta & consta \\
\hline Lista de ingredientes obrigatórios & consta & consta & consta & consta \\
\hline Conteúdos líquidos & consta & consta & consta & consta \\
\hline Identificação da origem & consta & consta & consta & consta \\
\hline Identificação do lote & consta & consta & consta & consta \\
\hline Prazo de validade & consta & consta & consta & consta \\
\hline Declaração da água na lista de ingredientes & consta & consta & consta & consta \\
\hline Declaração dos aditivos na lista de ingredientes & não consta & consta & consta & consta \\
\hline
\end{tabular}

No que concerne à rotulagem, foi observada a presença de informações mínimas obrigatórias e verificados também os seguintes pontos: a) todas as marcas designaram corretamente o produto, conforme preconizado pela legislação vigente ${ }^{[6]}$, mas apenas as marcas C e D dispuseram esta informação no painel principal da rotulagem de acordo com o que rege a legislação; b) a advertência obrigatória: "Crianças, gestantes, nutrizes, idosos e portadores de enfermidades: consultar o médico antes de consumir 
este produto", esteve presente em todas as marcas, mas apenas a marca $\mathrm{D}$ atendeu simultaneamente a exigência de registrar em destaque e negrito esta informação; c) todas as marcas apresentaram a lista de ingredientes obrigatória em ordem decrescente de proporção; d) a declaração dos aditivos é obrigatória na lista de ingredientes, mais a marca $\mathrm{A}$ não apresentou conformidade quanto à posição desta declaração na lista. Conforme a legislação ${ }^{[6,18,19]}$ em vigor, os aditivos alimentares devem ser declarados depois dos ingredientes.

\section{CONCLUSÕES}

Os parâmetros químicos e físico-químicos de identidade e qualidade dos compostos líquidos pronto para o consumo analisados estavam de acordo com a legislação brasileira em vigor, com exceção da concentração de vitamina $\mathrm{C}$, na marca $\mathrm{D}$, que se apresentou superior ao limite máximo permitido.

Tendo por base a avaliação dos rótulos é possível concluir que, a totalidade das marcas analisadas não atendeu integralmente aos requisitos de rotulagem exigidos pela legislação em questão, apresentando somente informações mínimas obrigatórias. Devido este fato, podemos dizer que é de extrema importância, a apresentação de todas as informações na rotulagem de um produto, especialmente para a segurança do consumidor.

\section{REFERÊNCIAS}

[1] Ballistreri MC, Corradi-Webster CM. O uso de bebidas energéticas entre estudantes de educação física. Rev LatinoAm Enfermagem. 2008;16(especial):558-564.

[2] Brasil. Ministério da Saúde. Agência Nacional de Vigilância Sanitária. Portaria no 222, 24 de março de 1998. Dispõe sobre regulamento técnico para fixação de identidade e qualidade de alimentos para praticantes de atividade física. Diário Oficial da União, Brasília, 25 mar. 1998. Seção 1, p. 13.

[3] Maughan RJ. Nutritional ergogenic aids and exercise performance. Nutr Res Rev. 1999;12(1):255-280.

[4] Juhn MS. Popular sports supplements and ergogenic aids. Sports Med. 2003;33(12):921-939.

[5] Maughan RJ, King DS, Lea T. Dietary supplements. J Sports Sci. 2004;22(1):95-113.

[6] Brasil. Ministério da Saúde. Agência Nacional de Vigilância Sanitária. Resolução. RDC no 273, 22 de setembro de 2005. Dispõe sobre regulamento técnico para misturas para o preparo de alimentos e alimentos prontos para o consumo. Diário Oficial da União, Brasília, 23 set. 2005. Seção 1, p. 375.

[7] Graham TE, Rush JW, Van Soeren MH. Caffeine and exercise: metabolism and performance. Can J Appl Physiol. 1994;19(2):111-138.

[8] Graham TE. Caffeine and exercise: metabolism, endurance and performance. Sports Med. 2001;31(11):785807.

[9] Graham TE. Caffeine, coffee and ephedrine: impact on exercise performance and metabolism. Can J Appl Physiol. 2001;26(1):103S-119S.

[10] Nehlig A, Daval JL, Debry G. Caffeine and the central nervous system: mechanisms of action, biochemical, metabolic and psychostimulant effects. Brain Res Rev. 1992;17(2):139-170.

[11] Spriet LS. Caffeine and performance. Int J Sport Nutr. 1995;5(1):84S-99S.

[12] Prada DB. Comentários sobre las llamadas bebidas energizantes, Servicio de Toxicologia del Sanatorio de niños. 2005 [acesso em 28 fev 2009]. Disponível em: http://www.sertox.com.ar/modules.php?name=Content\&p $\underline{a=\text { showpage\&pid }=238}$

[13] Universia. Avanza el consumo de energizantes com alcohol, 2008 [acesso em 31 jan 2008]. Disponível em: http://www.universia.com.ar/materia/materia.jsp?materia $=2$ $\underline{5768}$

[14] Ferreira SE, Mello MJ, Formigoni MLOS. O efeito das bebidas alcoólicas pode ser afetado pela combinação com bebidas energéticas? Um estudo com usuários. Rev Assoc Med Bras. 2004;50(1):48-51.

[15] Instituto Adolfo Lutz. Normas Analíticas do Instituto Adolfo Lutz: Métodos químicos e físicos para análises de Alimentos. 3o ed. São Paulo; 1985.

[16] Pearson D. Técnicas de laboratório para el análises de alimentos. Zaragoza: Acríbia; 1976.

[17] Brasil. Ministério da Agricultura, Pecuária e Abastecimento. Instrução Normativa no 24, 08 de setembro de 2005. Dispõe sobre o manual operacional de bebidas e vinagre. Diário Oficial da União, Brasilia, 20 set. 2005. Seção 1, pág. 11.

[18] Brasil. Ministério da Saúde. Agência Nacional de Vigilância Sanitária. RDC no 259, 20 de setembro de 2002. Dispõe sobre regulamento técnico sobre rotulagem de alimentos embalados. Diário Oficial da União, Brasília, 23 set. 2002. Seção 1, p. 33.

[19] Brasil. Ministério da Saúde. Agência Nacional de Vigilância Sanitária.RDC no 360, 23 de dezembro de 2003. 
Dispõe sobre regulamento técnico sobre rotulagem nutricional de alimentos embalados, tornando obrigatória a rotulagem nutricional. Brasília: Diário Oficial da união, Brasilia, 26 dez. 2003. Seção 1, p. 33.

[20] Fonseca JS, Martins GA. Curso de Estatística. 6a ed. São Paulo: Atlas, 1998.

[21] Fontes LCB. Uso de solução conservadora e de películas comestíveis em maças da cultivar Royal Gala minimamente processada. Efeito na fisiologia e na conservação [dissertação]. Piracicaba: Escola Superior de Agricultura Luis de Queiroz, Universidade de São Paulo; 2005.

[22] Asenjo CF. La ciência moderna: aspectos químicos para nutritivos da la acerola (Malpighia punicifolia L.). Ciência Revista Hispano Americana de Ciências Puras y Aplicadas. 1959:19(6/7):109-119.

[23] Chitarra AB, Alves RE. Tecnologia de pós-colheita para frutos tropicais. Fortaleza: Frutal - Sindifruta, 2001.

[24] Prestámo G, Manzano P. Peroxidase of setected fruits and vegetables and the possible use of ascorbic acid as an antioxidant. Hortscience. 1993;28(1):48-50. 